\title{
THE SIGNIFICANCE OF BIOLOGIC REACTIONS IN SYPHILIS OF THE CENTRAL NERVOUS SYSTEM
}

\author{
With NOTES ON TREATMENT, ESPECIALly INTRASPINAL * \\ DAVID J. KALISKI, M.D., AND ISRAEL STRAUSS, M.D. \\ NEW YORK CITY
}

In a paper published in 1918 , we ${ }^{1}$ reported an intensive study of this subject in the neurologic wards and serologic laboratory of the Mount Sinai Hospital. We now desire to bring our experiences down to date and to express our present views. In the light of a tendency to overemphasize the importance of early spinal fluid reactions, we have deemed it expedient critically to review these reactions and to estimate their significance as regards changes in the nervous system, and their proper relation to the nature and intensity of treatment.

Our knowledge of cytodiagnosis of the cerebrospinal fluid began with the work of Widal, Ravaut and Sicard ${ }^{2}$ in 1900 . The addition of the Wassermann test, protein estimation and the colloidal gold test placed the diagnosis of syphilis of the central nervous system on a definite basis.

PRECLINICAL CHANGES IN SYPHILIS CF CENTRAL NERVOUS SYSTEM

Ravaut showed that during the primary and secondary stages of syphilis, there is in 68 per cent. of the cases, a latent nonsymptomatic meningitis, the only evidence of which is obtained by lumbar puncture. Dreyfus $^{3}$ found similar changes in almost 80 per cent. of the cases. These figures have been corroborated by numerous observers. Perhaps if puncture were performed often enough, such changes might be found in all early cases. The departure from the normal in the early stages consists principally of increase in the cell count, protein increase (albumin of globulin; phase 1 of Nonne), and occasionally, of the presence of the Wassermann reaction. These facts are incontrovertible; it is their interpretation and significance as indicating permanent or ephemeral change in the neuraxis that are in question.

* From the Neurologic Service of the Mount Sinai Hospital, New York.

* Read before the Section on Nervous and Mental Diseases, at the SeventySecond Annual Session of the American Medical Association, Boston, June, 1921.

1. Kaliski, D. J., and Strauss, I.: On Syphilis of the Nervous System: Pathological, Serological and Clinical Criteria with Especial Reference to Treatment, Am. J. Syphilis 2: No. 4, 1918.

2. Widal, F.; Sicard, A., and Ravaut, P.: Bull. Soc. méd. d. hôp de Paris, 18:31-34, 1901; Ann. de dermat. et syph. 4:1-14, 1903; Thèse de Paris, 1899.

3. Dreyfus, G. L.:Deutsch. med. Wchnschr. 45:1293-1326, 1919; München med. Wchnschr. 66:1374-1376, 1919. 
According to Ravaut, ${ }^{4}$ the early changes are transitory and of the nature of a septicemia, while those occurring later in the disease are definitely focal, usually of the nature of a meningovasculitis. These latent reactions are common up to the fourth year, as shown in the chart, diminishing from that time to the twelfth year, and remaining more or less stationary up to the twentieth year, when they again diminish. Persistence of the septicemic reaction after the third year is, in the opinion of Ravaut, evidence of involvement of the central nervous system, especially if all the reactions are positive (the number of cells, the globulin and Wassermann reaction). Thus, lumbar puncture

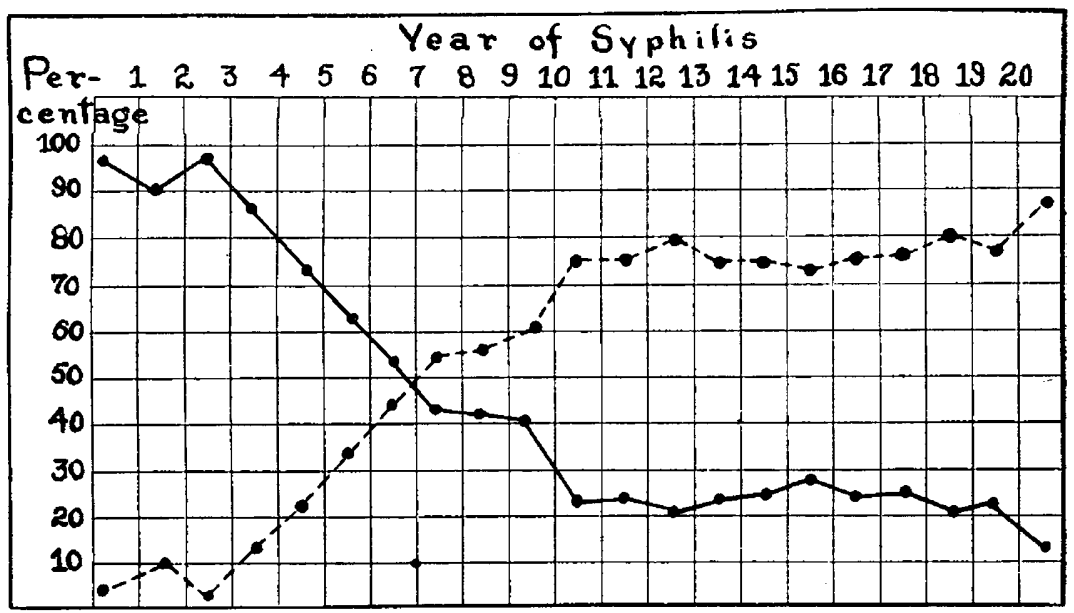

Relationship between biologic phenomena in spinal fluid and neurosyphilis (after Ravant).

Continuous line indicates presence of biologic changes in fluid and absence of clinical signs of neurosyphilis. Broken line indicates definite neurosyphilis accompanied by spinal fluid changes.

should be practiced during the first three years following infection, and if not then, at least at some period between the fourth and the tenth years, because then the ephemeral septicemic reactions tend to disappear while the clinical symptoms both objective and subjective tend to increase steadily. The importance of lumbar puncture is stressed by Ravaut as affording the only means of determining the presence of the latent meningitic condition, thus enabling one to take measures to eradicate it. To obtain evidence of the presence of syphilis in nervous or psychic disorders, especially if the blood reactions are negative, it is imperative.

Since we lack precise knowledge of the morphologic changes in early syphilis of the central nervous system, numerous hypothetic explanations have been offered for the presence in the spinal fluid of

4. Ravaut, P.: Presse méd. 1919, No. 57. 
increased cells and protein and for the Wassermann reaction. According to Gennerich, ${ }^{5}$ these changes are due to a histologic neurorecidive, and are found up to the third year in from 40 to 60 per cent. of early cases. Gennerich believes that the degree of change in the pia-arachnoid determines whether there will be eventual changes in the neuraxis of a metasyphilitic type, and he assumes that the pia-arachnoid serves as a barrier between the cerebrospinal fluid and the neuraxis. If the piaarachnoid is pathologic, fluid diffusion occurs into the nervous tissue with the occurrence of pathologic changes.

Wechselmann, ${ }^{6}$ in 1912 , reported changes in the cerebrospinal fluid in the preroseolar period of syphilis. Plaut, ${ }^{7}$ in considering the question of nervous involvement in early syphilis, speaks of the neurotropism of strains of spirochete, a belief engendered principally by the work of Fournier, Levaditi and Marie, ${ }^{8}$ and concurred in by many workers, including Nichols and Hough, ${ }^{9}$ and Reasoner. ${ }^{10}$ Plaut describes the finding of spirochetes in the cerebrospinal fluid of patients with early syphilis, but is doubtful if this is the beginning of syphilis of the central nervous system. He says there is no proof that the presence of organisms in the fluid has any relation to late syphilis of the nervous system, since most of the cellular reactions disappear from the fluid spontaneously or as a result of early treatment. Both Gennerich and Mulzer have reported cases showing spirochetes in the fluid with negative biologic reactions. Wile and Hasley ${ }^{11}$ believe that the so-called early preroseolar meningeal involvement which they found in forty-nine of two hundred and twenty-one cases "is for the most part transitory and without subsequent or permanent damage because in the majority the fluid was rapidly restored to normal under the influence of treatment and doubtless might have disappeared even without this treatment, like the early rash of syphilis." According to these authors, the appearance

5. Gennerich, W.: Berlin, in 1913; Hirschwald: Die Liquorveränderungen in den einzelnen Stadien der Syphilis, Deutsch. med. Wchnschr. 44:1243, 1918.

6. Wechselmann, W.: Deutsch. med. Wchnschr. 38:1446, 1912.

7. Plaut: Deutsch. med. Wchnschr. 1919, No. 48; Ztschr. f. d. ges. Neurol. u. Psychiat. 52:193, 1919; Neurotherapy, 1:76-88, 1919; 74:988, 1920.

8. Levaditi, C., and Marie, A.: Rev. de méd. 37:193, 1920.

9. Nichols, Henry J., and Hough, William H.: J. Exper. Med. 19: No. 4, 1914; Demonstration of Spirocchaeta Pallida in the Cerebrospinal Fluid, J. A. M. A. 60:108 (Jan. 11) 1913.

10. Reasoner, M. A.: Some Phases of Experimental Syphilis, J. A. M. A. 67:1799-1805, 1916; Early Death from Cerebral Syphilis, with Successful Rabbit Inoculation, ibid, 66:1917 (June 17) 1916.

11. Wile, U. J., and Hasley, C. K.: Involvement of Nervous System During Primary Stage of Syphilis, J. A. M. A. 76:8 (Jan. 1) 1921; Serologic Cure (?) in the Light of Increasingly Sensitive Wassermann Tests, ibid. 72:1526 (May 24) 1919. 
of positive fluid findings before the Wassermann reaction in the blood becomes positive is evidence of generalization and not of neurotropism.

Stokes, ${ }^{12}$ on the other hand, declares that early pleocytosis means involvement of the central nervous system in the sense of other tissue involvement in early syphilis. According to Dreyfus, ${ }^{3}$ an observer of extensive experience, pathologic changes in the fluid indicate infection of the neuraxis. He believes that these can recede spontaneously only in the early stages. He found changes in 80 per cent. of his cases in the early stage, but includes as evidence of pathologic change, pressure above $180 \mathrm{~mm}$. of mercury, to which we ascribe little or no importance. Fordyce ${ }^{13}$ says the "activity of a syphilitic process in the central nervous system is indicated much earlier, more accurately and often only by the cytologic changes in the spinal fluid which should always supplement the physical examination."

Observers both here and abroad differ in the quantitative interpretation of spinal fluid findings. Thus, Cornaz ${ }^{14}$ speaks of 2.4 cells per cubic millimeter as normal. Wile and Hasley ${ }^{11}$ set an arbitrary normal of 8 cells and state that an increase of one or two cells can hardly be accepted as definite proof of involvement. Dreyfus ${ }^{3}$ considers more than 5 cells as pathologic. Fildes, Parnell and Maitland ${ }^{15}$ refuse to accept the high percentage of involvement published by Ravaut in view of the fact that abnormality is often based solely on the finding of 10 cells per cubic millimeter. They state that few of these cases show any abnormal signs such as would produce disability and many show no discoverable signs of disease.

Obviously, we need a standard of serologic normality, and this we have attempted to determine. We have examined thousands of fluids from patients with and without demonstrable involvement of the central nervous system from all types of specific and nonspecific disease, and we have come to look on from 8 to 10 lymphocytes per cubic millimeter as within the bounds of normality. These figures may appear too high, but we believe it safer to set a standard which, if exceeded, in all probability means meningitic change. Indeed, a slight increase above this normal in the absence of clinical signs or symptoms, unless accompanied by definitely increased globulin or by a positive Wassermann reaction, is probably of little significance. Persuns without change in the central nervous system on whom spinal puncture is performed as a routine for general diagnosis, occasionally show as high as from 8 to 10 cells without globulin increase or other pathologic changes. On the other hand, definite syphilitic cases often show no increase in cells.

12. Stokes, J. H.: M. Clinics N. America 2: 1919.

13. Fordyce, J. A.: Am. J. M. Sc. March, 1921

14. Cornaz, Georges: J. Nerv. \& Ment. Dis. 49:282 (April) 1919.

15. Fildes, Parnell and Maitland: Brain 41:255 (Nov.) 1918. 
Cases of nonsyphilitic disease of the nervous system, such as multiple sclerosis, tumors of the cord and brain, chorea, epilepsies and other conditions frequently show 5 or more cells to the cubic millimeter without demonstrable meningitic change. ${ }^{16}$ It is well to bear in mind that pleocytosis is often rapidly influenced by puncture only or antispecific treatment-a drop to normal limits from a fairly high count not being infrequent.

Increased protein content has always been looked on as evidence of organic change. It is supposed to be the earliest sign to appear and the last to disappear either spontaneously or as a result of treatment. On the whole, it may be said to go hand in hand with cell increase. It may be the only abnormal sign present in the fluid. By itself, globulin increase is of little significance in syphilis of the nervous system, and, unless looked on as a corroborative sign of other biologic phenomena, could be dispensed with in routine diagnosis. ${ }^{17}$ A positive Wassermann reaction is of more significance, especially if the reaction is present in all dilutions from 0.1 c.c. upward. We have adopted 1 c.c. of fluid as the maximum amount. In the earliest stages of syphilis a positive reaction with large amounts of fluid is occasionally a transient phenomenon and may possibly be due to a transference of syphilitic reagin through the choroid plexus or other secreting surfaces of the piaarachnoid. A persistently positive Wassermann reaction of the fluid, when present in early syphilis with pleocytosis and globulin increase, especially if the reaction is present in all dilutions, even in the absence of clinical symptoms referable to the central nervous system, should be looked on with suspicion, and calls for persistent treatment and lumbar puncture from time to time in the control of the therapy.

We consider the colloidal gold test of little value in early syphilis. In neurosyphilitic conditions it may serve to corroborate the other tests, but may be found in the absence of all other phenomena. As an indication of the eventual development of paretic changes, the so-called paretic curve should not be relied on. (We do not believe the paretic curve is diagnostic of general paresis, even though most paretic fluids give it.) In other words, given an early case of syphilis with serologic reactions persistently positive, even with a positive blood reaction and the so-called paretic curve, we believe that we cannot today prognosticate the development of parenchymatous syphilis.

16. All counts should be made within an hour or two of puncture. A standard chamber should be used-the Fuchs-Rosenthal. If microscopic blood is present, the number of cells to an equivalent field should be noted. Frankly bloody fluids are not available for counts.

17. In estimating globulin increase it must be emphasized that only those fluids should be tested which are microscopically free from blood or contain at most a few red cells to the cubic millimeter. 
In short, we believe that a mere increase in cells (pleocytosis) and in protein (globulin) with a transient Wassermann reaction point to irritation of the pia-arachnoid of the nature of a general septicemic reaction, temporary, and of doubtful significance as to the future involvement of the neuraxis. ${ }^{18}$ The constant presence of cells, globulin and a definite Wassermann reaction in all dilutions in early syphilis indicate a more serious membrane involvement, possibly the earliest signs of a definite organic involvement of the neuraxis. ${ }^{19}$ This view is tentative despite the fact that we have observed cases of syphilis from their incipiency with persistent serologic reactions with no subjective or objective clinical manifestations, over a period of ten years. The literature is curiously lacking in adequate statistical studies of cases with such persistent serologic reactions (serologic neurosyphilis) which have eventually developed definite involvement of the central nervous system.

It is a fact to remember that patients with vascular neurosyphilis may show no change in the cerebrospinal fluid-occasionally the blood Wassermann reaction is positive--but may suffer from serious damage due to vessel closure or rupture. These patients often are young people with early syphilis. They must be borne in mind when interpreting biologic data and when forming a prognosis based on tests of the cerebrospinal fluid.

\section{INFECTION OF CENTRAL NERVOUS SYSTEM}

One of the most important questions to be considered is the time of infection of the central nervous system. Morphologic studies are entirely lacking to decide this point. The biologic reactions are not conclusive. The idea that spirochetes enter the central nervous system in the early stages of the infection or not at all, and lie dormant in

18. If the early spinal fluid changes found in so high a percentage of syphilitic patients were indicative always or even frequently of invasion of the central nervous system by the spirochete, it is difficult to understand why symptomatic syphilis of the central nervous system is not more frequently encountered. We know that the neuraxis as a rule is susceptible to slight irritation or invasion by virulent organisms, and the nervous system in early syphilis should therefore in the circumstances respond with productive or other types of inflammation to an invader like the spirochete at a stage when the germ possesses its maximum virulence and the body resistance is at its lowest.

19. Fraser, A. R.: Am. J. Syphilis, 5: No. 2, 1921. Fraser believes that the presence of serologic reactions in the fluid in the absence of clinical signs of involvement of the central nervous system points to the presence of an ample antibody supply with protection of the neuraxis, while the negative reaction means that the central nervous system has escaped involvement or that it has failed to react, which, he cannot tell. He believes that the positive early reactions in the fluid mean that the nervous system has reacted and therefore the prognosis is better. 
relatively nonvascular regions but possess the potential power of later multiplication and assumption of virulence in response to some unknown stimulus, is still unproved. Is it not possible that spirochetes wander into the central nervous system from without and, finding a less resistant or more fertile soil, become active? ${ }^{20}$ If spirochetes do lie latent in the neuraxis for years, as stated by Warthin, ${ }^{21}$ what is responsible for this so-called immunity or failure to react? If immunity exists in syphilis, it has not been proved by the demonstration of immune bodies, antibodies, in the blood or spinal fluid. Clinically, soon after the chancre, even before the generalization of the virus, the body becomes insusceptible to reinoculation with syphilis and in the vast majority of cases remains so. Even in a patient with a case of syphilis of long standing when the "immunity" has become attenuated, if it ever does so, reinfection is rare, even after years of therapy and with absolutely negative serologic reactions, so that when we speak of immunity in syphilis, we are using an empiric term, not one scientifically exact.

We speak of antibodies produced in response to syphilitic infection being responsible for so-called immunity in syphilis, or of protective antibodies being present in the blood and spinal fluid, detectable by the Wassermann reaction. All this is hypothetical. There is no proof fulfilling immunologic requirements. We know that the antigen of the Wassermann reaction is of lipoidal nature and that the spirochete itself cannot thus be practically utilized; further, other protozoan diseases definitely not syphilitic give a high percentage of positive Wassermann reactions. Wassermann ${ }^{22}$ himself has recently shown that there is present in the serum of syphilitic patients a substance that in the presence of complement enters into a reversible combination with the Wassermann antigen. The extract does not consist of the disease producing spirochetes or other protein material, but of alcohol-soluble, fat-like substances (lipoids) which are derived from animal organs.

20. Brown and Pearce showed that rabbits which recovered from clinical manifestations of syphilis are still infected and may continue to harbor virulent spirochetes in certain lymph nodes. The points of rest during latency are possibly chiefly the lymphoid tissues of the animal. Arzt and Kerl Wien. klin. Wchnschr. 27:29 (July 16) 1914, reported finding in lesions about the genitals and in the regional lymphnodes of normal rabbits spirochetes absolutely identical morphologically with Spirochaeta pallida. These organisms were found in 26.9 per cent. of certain strains of normal rabbits. These observations have been confirmed by Jakobsthal, by Tomascewski, and in this country by Brown. These findings point out the necessity for great conservatism in accepting the conclusions drawn from rabbit inoculations and in our estimation call for a complete revision of all prior experimental inoculation work in rabbits.

21. Warthin, A. S. Am. J. M. Sc. 152:508, 1916.

22. Recent experimental investigations on syphilis, Berlin letter, J. A. M. A. 76:463, 1921. 
This substance, which Wassermann recently isolated for the first time, is produced by the lipoid substance which must be present in large quantities in the blood of syphilitic patients. Wassermann attempts to prove a point heretofore unknown, namely: that lipoids as well as protein substances may produce genuine antibodies, thus explaining why the so-called antibodies present in syphilis were directed not against the specific spirochete of the disease, but against lipoids produced by spirochetal action on the tissue cells of the body. He asserts that the syphilitic patient thus suffers from an inversion of lipoid metabolism. ${ }^{23}$

In a recent paper Fraser ${ }^{24}$ speaks of increase of neurosyphilis due to intensive treatment by arsphenamin in early syphilis after generalization causing rapid sterilization with loss of antibody protection for the central nervous system. He thinks that because of a lack of vigorous cell reaction, the spirochetes penetrate into relatively nonvascular areas of the central nervous system; that the central nervous system produces few or no antibodies and is dependent for protection on those produced as a result of the early general reaction. This is only a restatement of the views of Gennerich, ${ }^{5}$ who holds that treatment, especially if insufficient, during the period of early dissemination, hinders generalization of the spirochete and causes lack of immunity. Spontaneous cure of the early disease does not destroy this early ample immunologic response, and the stronger the immunity the fewer the neurorecidives (meningitic or other). Local foci of spirochetes develop in the presence of a lesser immunity caused by insufficient treatment or when generalization is hindered. In other words, by means of insufficient early treatment or by a restriction of early immunity formation we have instead of the sterilisatio magna of Ehrlich, a sterilisatio fere completa. These views are combated by Matzenauer, ${ }^{25}$ according to whom the severity and type of lesions are explainable on the basis of constitutional factors and individual susceptibility.

If immunity exists in syphilis, as is claimed because of the long period of incubation and invasion, the protracted latent period and various other phenomena, such as rare reinfection nonautoinoculability, we have as yet no scientific proof of this hypothesis.

23. Brown, W. H., and Pearce, L.: J. Exper. M. 31:749, 1920; 33: (May 1) 1921 ; Am. J. Syphilis, 5:1, 1921. In a series of rabbit experiments these authors have shown that in animals inoculated intratesticularly with virulent strains of spirochetes, those given one injection of arsphenamin and subsequently reinoculated after the disappearance of early clinical symptoms, developed typical reinfections in the shape of a second chancre, while the untreated infected controls failed to react a second time. By these experiments they attempt to prove that even in uncured syphilis, reinfection is possible if the resistance is lowered by insufficient "immunity" reaction.

24. Fraser, A. Reith: Am. J. Syphilis 5: No. 2, 1921.

25. Matzenauer, R.: Wien. klin. Wchnschr. 32:831, 1919. 
The question of neurotropism of spirochetes may be merely glanced at. Some believe that certain strains have an elective affinity for the nervous system. In spite of the extensive animal work of Levaditi and Marie, ${ }^{8}$ of numerous assertions of the rarity of nervous involvement among negroes, of cases of conjugal neurosyphilis, of the variation in morphologic appearances of certain types of spirochetes and of numerous instances of neurosyphilis in persons infected at the same source, we hold with Sicard, ${ }^{26}$ Nonne ${ }^{27}$ and a host of others that actual proof of the presence of special strains of the spirochete is as yet lacking.

Among our patients, and others have made similar observations, are persons infected at the same source, some of whom developed nervous lesions while their partners did not.

TREATMENT OF SYPHILIS OF THE CENTRAL NERVOUS SYSTEM

Our own results in the eradication of clinical and biologic signs in the first year following infection with almost complete prevention of serious invasion of the central nervous system when treatment has been intensive enough, leads us to believe in the institution of therapy at the earliest possible moment and of such an intensity as is compatible with the patient's welfare. The proper care of a patient with syphilis of the nervous system demands that the physician be conversant with modern laboratory diagnosis, equipped to evaluate the biologic data, able to make a detailed neurologic examination and especially capable of reaching a logical and practical conclusion after digestion of all the evidence.

We strongly believe in the efficacy of combined treatment in most forms of syphilis of the central nervous system, with certain notable exceptions. We feel sure it is wise to adapt the treatment to the patient in the more chronic types of syphilis of the central nervous system rather than to carry out a stereotyped plan designed to eradicate biologic phenomena of the disease.

Let us realize that neurosyphilis cannot be judged or classified by the outcome of biologic reactions alone, but that of equal, often greater, importance are the clinical factors. Absolutely essential is a knowledge of the possibilities and limitations of various forms of therapy; also, a broad grasp of the patient as a complex human being with a psyche, not merely as a bundle of biologic phenomena to be made negative. We wish to protest against a form of therapy that often has a mortality of 20 per cent. (intracranial therapy) with merely promising results,

26. Sicard, J. A.: Soc de neurol. de Paris, July 9-10, 1920; Presse méd. 28:52, 1920; 28:281, 1920; Médicine, Paris 2:3 (Nov.) 1920.

27. Nonne: Syphilis und Nervensystem, Berlin, 1915. 
and against all extra-hazardous and painful forms of therapy that do not offer more than a debatable chance for even prolongation of life, not to speak of cure.

Examinations of blood and spinal fluid in the so-called preclinical stage of syphilis of the central nervous system are of great importance. The case of the patient with fully developed neurosyphilis of any type is different. At this stage objective clinical signs are present and laboratory data must be looked on as corroborative, even though occasionally in the presence of dubious or merely suspicious clinical evidence, laboratory data may be the determining factors.

Treatment of Patients with Acute Cases.-In our previous paper we divided the cases clinically into three groups. In the first we placed all urgent cases with acute symptoms requiring immediate and intensive treatment, as well as all early cases of cerebrospinal syphilis of any type, early tabes and cases of general paresis. Toward these cases we have gradually assumed a more conservative attitude. We no longer follow the plan of Dreyfus, and we rarely give intravenous medication oftener than twice a week-more frequently only every five days until urgent symptoms are abolished, when the case is placed in Group 2. which includes the more chronic types of cerebrospinal syphilis, tabes and certain cases of general paresis. We rarely exceed $0.4 \mathrm{gm}$. of arsphenamin or its equivalent. The first injection we make minimal to avoid a Herxheimer reaction. In involvement of the medulla, optic or other cranial nerves, if time permits, we precede the injection of arsphenamin by one or two of a soluble mercurial (mercuric chlorid or succinimid) to prevent too intensive action by the arsenical. During the administration of any antisyphilitic in acute medullary involvement, the pulse and respiration are carefully guarded from the too intensive action of any spirocheticidal remedy. In the more chronic group, arsphenamin is given not oftener than once a week and in doses ranging from 0.25 to $0.4 \mathrm{gm}$., never higher. We believe that the spirocheticidal remedies are of no value if they exert their toxic action. We aim to get the tonic action of the arsenicals for chronic sufferers from specific nervous disease.

General Paresis.-As regards general paresis, we are convinced of the futility of intraspinal and intracranial therapy, which we unhesitatingly condemn. Well developed cases treated in groups for comparison did as well on one type of treatment as on another. ${ }^{28}$ All eventually succumb. The tendency of dementia paralytica to sudden remissions, even intermissions, must always be borne in mind. Patients

28. Darling, I. A., and Weston, P.: Penn. M. J. 22 (March) 1919 (discussion) Jackson, J. A., and Pike, H. V.: Interpretation of Wassermann Reaction of Blood Serum in Mental Diseases, J. A. M. A. 76:360 (Feb. 5) 1921. 
with early cases frequently are astonishingly benefited by intravenous injections of arsphenamin, combined with injections or inunctions of mercurials, and exhibit prolonged remissions during which it is often quite impossible to detect any signs of mental aberration. The diagnosis of general paresis may offer exceptional difficulties even in the presence of positive biologic reactions in the blood and spinal fluid. In the absence of definite mental symptoms there has been a growing tendency to call these cases "serologic" paresis, paresis sine paresi. ${ }^{29}$ The criteria of Darling ${ }^{30}$ for true paresis are: speech disorders, pupillary changes, reflex changes, amnesia, quick shifting emotions, character changes and conduct slump. In the hope of a prolonged period of remission or that possibly what we believe to be paresis may prove to be a more hopeful cerebral syphilis, we practice persistent treatment of these cases.

Lumbar Puncture.-This brings up the question of lumbar puncture. We believe that on every patient with early syphilis lumbar puncture should be performed at least once, preferably at the end of the first year of treatment and earlier if indicated by clinical evidence of involvement of the nervous system. If the result is negative, it need not be repeated, if treatment is continued, until the third or fourth year. If then negative, in the absence of clinical signs of nervous syphilis, we do not repeat it ( see plan). If positive, we do not repeat it oftener than once a year during the continuance of therapy. In latent cases or in late cases of syphilis with any suspicion of nervous or mental change, of course we perform lumbar puncture as a diagnostic measure. During treatment, we believe that a great deal of harm may be done by too frequent tappings. We are guided by the clinical symptoms and the blood reaction, reserving puncture for the completion of treatment. As therapeutic indications we are guided first by the clinical symptoms and objective signs, to a lesser degree by the outcome of the tests. We are not inclined to agree to the performance of puncture in the outpatient department or consulting room, but insist on at least one day's rest in bed immediately following tapping

Résumé of Treatment of Syphilis of the Central Nervous System.-Intraspinal Therapy: A review of the literature has shown a growing tendency in this country to restrict intraspinal treatment to very narrow limits which has been largely due to the efforts of Sachs and his associates. In Europe with few exceptions there has been a general abandonment of intraspinal methods.

Our former opinion, which we still hold, of the very limited value of intraspinal treatment was based on these facts:

29. Southard and Solomon: Boston M. \& S. J. 24:1.

30. Darling, I. A. : Am. J. Syphilis 3:35, 1919. 
1. The amount of arsenic present in auto-arsphenamized serum was infinitesimal and of no spirocheticidal value.

2. There was no valid proof that arsphenamized serum was in itself spirocheticidal in vivo.

3. It was impossible to reinforce blood serum with more than an infinitesimal amount of arsphenamin.

4. The introduction directly into the cerebrospinal fluid of any of the arsenical preparations was often dangerous and occasionally exceedingly painful. When nontoxic quantities were used the remedy was impotent.

5. The pathologic changes in the various types of syphilis of the central nervous system are rarely only superficial, and are not reached by injection into the subarachnoid space.

6. The method was physiologically wrong. Nutrition of the cerebrospinal tissues is not afforded by the cerebrospinal fluid which serves as an hydrostatic agent for the suspension of the brain and cord and an avenue of excretion. Therefore, medication of the nervous tissues via the cerebrospinal fluid was impossible. Substances introduced into the cerebrospinal fluid are rapidly absorbed into the blood and rarely diffuse through the subarachnoid space. The direction of flow in the so-called perineuronal lymph space is away from the nervous tissue toward the cerebrospinal fluid. The pressure in the cerebral capillaries is greater than that of the cerebrospinal fluid so that fluid leaves the capillaries rich in material circulating in the blood, circulates in the pericapillary and perineuronal spaces, yielding nourishment and receiving waste matter, and finally leaves the tissues by the pericapillary and perivascular spaces to the subarachnoid cavities over the surface.

7. Arsenic injected intravenously regularly reached the cerebrospinal fluid in greater quantities than could possibly be injected intraspinally without damaging or destroying the nervous tissue. This is true also of mercury and iodids.

8. If the meninges are the seat of an acute or chronic inflammation, "antibodies" circulating in the blood stream reach the subarachnoid space. Intraspinal injections may serve to irritate the secreting mechanism and thus render it more permeable. Spinal drainage may act in a similar manner, as well as the reduction of fluid pressure.

If intraspinal therapy were a simple and harmless procedure, there would be little reason for an attempt to restrict its use. But we feel that the teaching of Fordyce and his followers is responsible for opening the door to a great deal of misguided and harmful effort due to lack of knowledge of the indications of this form of treatment, of failure to appreciate the true significance of the various biologic factors present in the disease, and above all, of insufficient experience in the various clinical manifestations of syphilis of the central nervous sys- 
tem. In our previous paper we showed fallacies in the conclusions of these therapists and we believe that time has shown the narrow limits for this form of therapy. ${ }^{31}$

In optic atrophy intraspinal therapy is not contraindicated, but we feel that the disease, if not too far advanced, may be as satisfactorily rendered stationary or retarded in its progress by intravenous therapy. It is questionable whether serum administered in the lumbar region ever reaches the region of the base near the origin of the optic nerves. In properly regulated dosage if preceded by mercury and reinforced by iodids, arsphenamin has no harmful effect on the optic nerves.

If we restrict the use of intraspinal therapy, as Fordyce ${ }^{32}$ has stated, to cases which after intensive treatment with arsphenamin, mercury and potassium iodid, show little or no improvement in symptoms, blood or fluid reactions, its field will be small indeed. It should by all means be a second rather than first choice in the treatment of neurosyphilis. Fordyce ${ }^{13}$ now advocates its use especially in the early invasive period when the biologic reactions persist in spite of intensive therapy. On the one hand, Fordyce advocates the removal of these biologic reactions by intraspinal and combined therapy, and on the other, McDonagh, ${ }^{33}$ Fraser, ${ }^{24}$ and others advocate their retention as an evidence of reaction on the part of the neuraxis with protective antibody formation, a sign of good omen. As we have shown, the importance of these early reactions as regards actual involvement of the central nervous system has been misunderstood by Fordyce and others. McDonagh denies the efficacy of intraspinal therapy because in spite of it, late degenerative lesions may occur. In our opinion, the mere presence of spinal fluid changes in early syphilis does not call for intraspinal therapy, because these signs are transient and do not, in the vast majority of instances, necessarily signify nervous changes and only quite exceptionally resist treatment by arsphenamin intravenously and mercury. Here again we are adverse to the use of intraspinal therapy, except in the very rare instances in which a few years of proper treatment have left unchanged all the biologic reactions in the fluid. ${ }^{34}$

In the following plan we have briefly summarized our methods of procedure in all stages of neurosyphilis, as well as in early constitutional syphilis.

31. What we believe these limits to be will be found in our article referred to in Footnote 1. Lack of space forbids their insertion here.

32. Fordyce, J. A.: Am. J. Syphilis 3:341, 1919.

33. McDonagh, J. E. R.: Venereal Diseases, London, 1920.

34. In addition to the references given, the following may be of interest: Sachs, B.: J. A. M. A. 69:681, 1917; Arch Neurol. \& Psychiat. 1:277-284, 1919.

Dercum, F. X.: The Functions of the Cerebrospinal Fluid with a Special Consideration of Spinal Drainage and of Intraspinal Injections of Arsphenamized Serum, Arch. Neurol. \& Psychiat. 3:230 (March) 1920. 
PLAN OF PROCEDURE IN SYPHILIS OF THE CENTRAL

NERVOUS SYSTEM

GROUP 1

Cases Requiring Immediate and Intensive Treatment:

Early cerebrospinal syphilis-at onset of or early in:

Meningitis

Meningomyelitis

Meningoencephalitis

Cerebral and spinal endarteritis (fresh insult)

Optic neuritis-advancing

Syphilitic epilepsy-earlier cases

Pseudo tabes

Ophthalmoplegias

Early tabes dorsalis-with acute symptoms, pains, crises, etc.

General paresis-with acute manifestations

Treatment-unless contraindicated :

1. Arsphenamin (or equivalent) 0.2 to $0.4 \mathrm{gm}$. every three to five days until symptoms are controlled, then $0.4 \mathrm{gm}$. every week-six to eight injections in each course. First course followed by treatment as in Group 2.

2. Mercury by deep intragluteal injections. Soluble salt every two to three days, alternating with above until symptoms demand less intensive treatment. Then follow injections by arsphenamin with weekly injections of insoluble preparations as in Group 2.

3. Iodids by mouth, if possible, rectally or intravenously otherwise.

4. Lumbar puncture for relief of coma, secondary hydrocephalus symptoms, root pains.

\section{GROUP 2}

More Chronic Cases-Later Stages of Group 1

Cases with persistent biologic reactions-general paresis :

1. Arsphenamin (or equivalent) 0.25 to $0.4 \mathrm{gm}$., once weekly or every ten days-six to eight injections followed by mercury.

2. Mercury, insoluble preparation every five days-or soluble preparation thrice weekly for two months or thirty rubbings.

3. Iodids by mouth, rectum or intravenously to point of tolerance for one month following mercury or alternating.

4. Sodium nucleinate intragluteal injections 0.3 to $1.0 \mathrm{gm}$. or more to induce febrile reaction-every 3 to 7 days-in general paresis and occasionally in resistant tabetic or spastic cases.

5. Reeducation-method of Maloney.

6. Symptomatic-baths, massage, medicinal for pain, bladder symptoms. Repeat once or twice during the first year as indicated by benefit derived, the condition of the patient's excretory organs, morale, biologic reactions, etc. Subsequently yearly or more or less often, gradually tapering off. See Group 3.

\section{GROUP 3}

Late Cases of Tabes Dorsalis, Cerebrospinal Syphilis, Optic Atrophy, Spastic Paraplegia, and Cases of Group 2 after Three Years: 
No attempt to influence biologic reactions, treatment mainly symptomatic and supportive.

1. Arsphenamin, 0.25 to $0.4 \mathrm{gm}$. every two weeks, not more than six injections in all; only if well tolerated.

2. Mercury by inunction or injection following No. 1 .

3. Iodids by mouth, following No. 2 .

4. Reeducation, method of Maloney.

5. Symptomatic, control of crises, bladder symptoms.

\section{AUTHORS' METHODS OF TREATMENT IN EARLY CONSTITU-}

TIONAL SYPHILIS

Treatment in Primary Syphilis:

Attempt at abortion of disease

Intensive intravenous arsphenamin therapy

Followed by mercury, rapid sterilization methods (Pollitzer, Scholtz)

Less intensive plans

Repetition of courses during first year.

Treatment of Secondary Syphilis-First Year :

(a) Attempts to eradicate symptoms.

(b) Attempts to eradicate biologic reactions: Arsphenamin intravenously every other day for three injections, then once weekly for five injections, followed by mercury twelve insoluble injections, twenty-four to thirty soluble injections. Rest from six to eight weeks, if symptomless, then arsphenamin weekly, for eight injections. Mercury, twelve insoluble injections, from twentyfour to thirty soluble injections. If Wassermann negative, rest for from two to three months. If Wassermann positive, repeat above treatment after from six to eight weeks' rest.

(c) Lumbar puncture: At the end of the first year, if the Wassermann reaction is negative and symptomless. At the end of second course, if the Wassermann reaction is positive or if there are symptoms of cerebrospinal involvement.

Treatment of Secondary Syphilis-Second Year:

(a) Symptomless-biologic reactions negative blood and cerebrospinal fluid-one course treatment-from four to six injections of arsphenamin, six injections of mercury.

(b) Symptomless-blood Wassermann reaction positive-cerebrospinal fluid negative. Two course treatment, six to eight injections of arsphenamin, twelve injections mercury insoluble, twenty-four to thirty injections of mercury soluble.

Treatment of Latent Syphilis :

After second year, Wassermann blood reaction positive.

1. Lumbar puncture to be advised. (a) Blood Wassermann reaction positive, cerebrospinal fluid negative. Treat as $(b)$ above. (b) Cerebrospinal fluid positive-blood positive. Treat as Group 1 for one course, rest six to eight weeks, then arsphenamin weekly for six injections followed by mercury.

2. Repeat each year as indicated by symptoms and presence of biologic factors. 


\section{DISCUSSION}

Dr. Walter F. Schaller, San Francisco: I have been interested in the pathogenesis of certain types of neurosyphilis and in the early diagnosis of this disease. My associate, Dr. Henry Mehrtens, has been occupied with studies of the cerebrospinal fluid, its pressure, circulation and absorption, and with the permeability of the meninges to drugs. Conjointly, along these lines, we believe to have found a justification for intraspinal treatment. In advanced cases, we can only hope for an occasional arrest and some symptomatic benefit. I believe that in certain forms of neurosyphilis, particularly in the meningeal form, such as tabes, we have obtained benefit when intravenous treatment has failed. In our experience the method is not dangerous although it requires a rigid aseptic technic. We give all our treatments in the hospital. There have been no more accidents in our series than in a corresponding number of arsphenamin injections alone. The intraspinal treatment includes, in addition to intravenous treatment, drainage and what additional benefit the application locally of specific medicines may have. The benefit has been both symptomatic and serologic. These patients return to us voluntarily, requesting further treatment, in spite of the physical discomfort and considerations of time and expense, because they are convinced that they have been helped.

Dr. Harry C. Solomon, Boston: Some patients do not respond well to intravenous treatment, but do respond very well to intraspinal therapy. These patients have developed essentially negative blood and spinal fluid findings and have improved their symptoms. We have treated our cases of neurosyphilis with intensive intravenous remedies for many months. In the last six months I have seen patients treated for three or four years with arsphenamin, mercury and iodids without effect, who after two, three, four or five intraspinal injections gave practically negative spinal fluid findings. There is some effect from intraspinal therapy, all theories to the contrary notwithstanding.

Dr. Harold E. Foster, Boston: Several cases of primary optic atrophy, (syphilitic), have been treated for a number of years at the Massachusetts General Hospital according to the Swift-Ellis method administered intracisternally. The first patient selected for this method had been under intravenous arsphenamin treatment for about a year with slowly steadily contracting fields. In about four months from the first intracisternal treatment of arsphenamized serum the fields were of the same size as in the first examination. The process had apparently been checked. About six months later the fields were expanded slightly. One other patient who has been faithful to treatment was greatly benefited. Most of these optic atrophy cases are sent us by the oculists after the disease has progressed rather far. If the neurologic departments could get these cases in the early stages, this intensive treatment would obviate these extreme conditions, but many patients come to us when they are only able to distinguish daylight from darkness. Without exception we have had results with the intracisternal arsphenamized serum administration which were not obtained by the intravenous route.

Dr. Henry F. Stoll, Hartford, Conn.: I have had patients who continued taking intraspinal treatments even though they had a reaction after each treatment because they felt better than after the intravenous treatments. I do not think that intraspinal treatments should be given in all cases, but when the symptomatic and serologic improvement is not satisfactory, they should be resorted to. Patients showing the paretic curve with a positive Wassermann reaction in a small amount of spinal fluid should be given the advantage of both intravenous and intraspinal therapy as soon as they come under observation. The paretic curve does not signify paresis necessarily, but it probably is indicative of serious parenchymal involvement.

DR. David J. KaliskI, New York: Our experience is based on the treatment of more than 500 cases by all methods of therapy. In the beginning when the Swift-Ellis method was first advocated we treated about 400 patients and some received as many as forty intraspinal injections. Occasionally, a patient 
improved clinically and serologically, but patients also improved after lumbar puncture and normal serum intraspinally; in the vast majority of cases also if intravenous treatment alone was properly carried out. We do not mean arsphenamin intravenously alone, but the use of mercury and iodids in proper sequence and dosage with mental and mechanical therapy to increase the patient's morale and improve his general condition. These adjuncts are just as important factors as any one "kind" of treatment, and it also explains why some patients who are taken from one kind of treatment and put on another do well; it is because they are stimulated, buoyed up by new hope of cure, and their general condition improves. Intraspinal therapy should not be used in every case. That is what we desire to emphasize. As far as the improvement of optic atrophy after puncture of the cisterna magna is concerned, Dr. Foster cited only one case. This is hardly sufficient to do more than draw attention to the possibilities of the method. As to the paretic curve with the colloidal gold reaction, Dr. Tilney found, and we agree with him, that in about 155 cases the paretic curve did not indicate parenchymatous syphilis. The important point is that the paretic curve by itself does not mean paresis. The recital of a series of cases benefited by a given form of therapy is not a fair way of deciding the comparative merits of that form of treatment. Intraspinal therapy by itself, no matter how intensively applied, is not as efficacious, on the whole, as intravenous therapy, to say nothing of the comparative risks and inconvenience and expense to the patient. In the use of arsphenamin in syphilis of the nervous system we must avoid maximum dosage. Rarely use more than $0.4 \mathrm{gm}$. arsphenamin and 0.6 neo-arsphenamin. This applies to the intensive treatment in the early stages or in the later cases. It is all-important to avoid getting a toxic effect from too large or frequent dosage and to avoid any form of treatment that tends to disturb the morale of the patient with syphilis of the nervous system. 